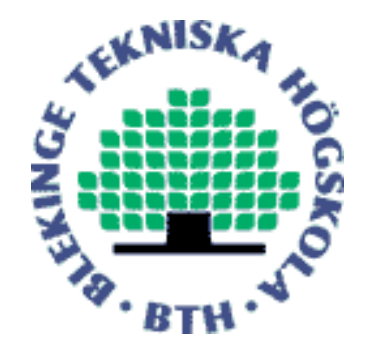

Copyright (C) 2008 IEEE. Citation for the published paper:

Josef Ström Bartunek, Mikael Nilsson, Jörgen Nordberg, Ingvar Claesson "Improved Adaptive Fingerprint Binarization"

CISP, 2008, Sanya, China

This material is posted here with permission of the IEEE. Such permission of the IEEE does not in any way imply IEEE endorsement of any of BTH's products or services Internal or personal use of this material is permitted. However, permission to reprint/republish this material for advertising or promotional purposes or for creating new collective works for resale or redistribution must be obtained from the IEEE by sending a blank email message to pubspermissions@ieee.org.

By choosing to view this document, you agree to all provisions of the copyright laws protecting it. 


\title{
Improved Adaptive Fingerprint Binarization
}

\author{
Josef Ström Bartůněk, Mikael Nilsson, Jörgen Nordberg, and Ingvar Claesson \\ Department of Signal Processing, School of Engineering, Blekinge Institute of Technology \\ Box 520, SE-372 25 Ronneby, Sweden \\ E-mails: jsb@bth.se,mkn@bth.se,jno@bth.se,icl@bth.se
}

\begin{abstract}
In this paper improvements to a previous work are presented. Removing the redundant artifacts in the fingerprint mask is introduced enhancing the final result. The proposed method is entirely adaptive process adjusting to each fingerprint without any further supervision of the user. Hence, the algorithm is insensitive to the characteristics of the fingerprint sensor and the various physical appearances of the fingerprints. Further, a detailed description of fingerprint mask generation not fully described in the previous work is presented. The improved experimental results are presented.
\end{abstract}

\section{Introduction}

Unprocessed grayscale fingerprint images usually contain unwanted artifacts caused by skin condition and sensor noise. For example, fingerprint smudges lifted from the crime scene by the forensic technicians are often very noisy. Enhancement and separation of fingerprints from the background denoted binarization is frequently employed as a preprocessing step in the fingerprint recognition systems to reduce the unwanted artifacts.

Contextual filtering is an interesting binarization category widely used in many algorithms. The basic concept of contextual filtering is to apply filters in local areas of the fingerprint aligned with the ridge orientation. Among the first methods designing directional filters are $[5,6]$. Others employs directional Gabor or Butterworth bandpass filters $[2,1]$. Second directional derivatives are exploited to construct the filters in [8]. Existing binarization methods among the ones mentioned above often lock various parameters such as having fixed local area or filter size. Hence, to achieve good binarization for arbitrary fingerprints each algorithm has parameters that needs to be tuned manually.

In previous work; these drawbacks ware addressed by proposing a novel binarization algorithm [3]. The new method is an entirely automated process adjusting itself to each fingerprint. Abilities such as automatically regulate size of the local area and adjustment of the directional filters demonstrates interesting adaptive behavior of the algorithm.

In this paper an additional post processing step is introduced eliminating excessive data in the binarized fingerprints improving the final result. Further, detailed description of fingerprint mask generation is presented together with its effective implementation.

\section{Previously proposed algorithm}

In this part a brief summary of our previously proposed fingerprint binarization algorithm is presented. The entire algorithm consists of three major parts. In the first part the fingerprint will be analyzed in order to estimate a suitable local area size containing multiple periods of ridges and valleys according to section 2.1. In the second part a detailed analysis of local areas in the fingerprint is performed according to section 2.2. In the last part a matched filters are designed and the binarization is carried out, see section 2.3.

The binarization algorithm is based on the 2-D Fast Fourier Transform (FFT) where its properties are important foundation in the algorithm. The FFT is a method that maps a signal from the spatial domain into the frequency domain. An important part of frequency domain analysis involves the periodogram [4]. The function

$$
\begin{array}{r}
f\left(n_{1}, n_{2}\right)=\cos \left(\omega_{p_{1}} n_{1}+\omega_{p_{2}} n_{2}\right) \\
0 \leq n_{1} \leq N_{1}-1 \\
0 \leq n_{2} \leq N_{2}-1
\end{array}
$$

is used to create a 2-D sinusoidal signal model, where $\omega_{p_{1}}$ and $\omega_{p_{2}}$ are found as the location of the highest peak in the magnitude centered spectrum denoted $\left|F\left(\omega_{1}, \omega_{2}\right)\right|$. Two sinusoidal signals and corresponding magnitude spectrums are illustrated in Fig. 1. A periodic signal generated with Eq. (1) is illustrated in a) and b). A part of real fingerprint is illustrated in c) and d). Many similarities can be observed by comparing the two signals and theirs frequency spectrums. It is interesting to observe that the dominant peak 


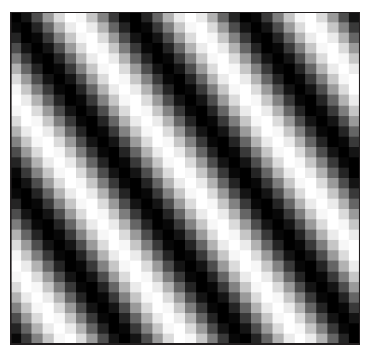

(a)

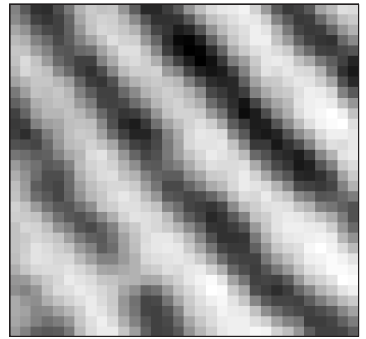

(c)

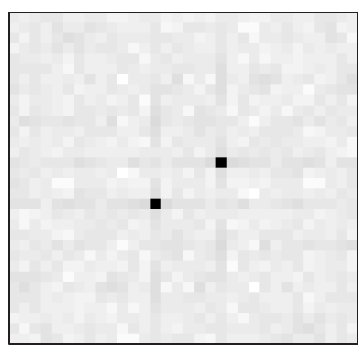

(b)

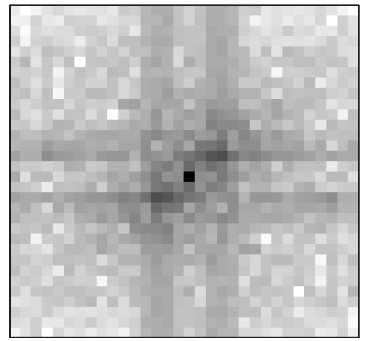

(d)
Figure 1. a) The periodic sinusoidal signal represented in the spatial domain; b) corresponding amplitude spectrums; c) Local area inside a fingerprint; d) the corresponding magnitude spectrum.

denoted $P_{D}$ in the magnitude centered spectrums are positioned at the same coordinates $\left(\omega_{1}, \omega_{2}\right)$. Hence, inserting the coordinates $\left(\omega_{1}, \omega_{2}\right)$ for the $P_{D}$ of the fingerprint into Eq. (1) will recreate the observed part of fingerprint as a periodic and even signal.

\subsection{Determining the Size of the Local Area}

The magnitude spectrum of the entire fingerprint contains a circular structure. By estimating the radius of the dominant circular structure the dominant spatial frequency in the fingerprint image can be found, see Fig. 2 a).

Due to the symmetry in the magnitude spectrum the calculations are performed in only one of the two regions. The average magnitude over all angles on the half plane for a given radius yields in a function

$$
A(r)=\frac{1}{\pi} \int_{0}^{\pi}|F(r \cos (\theta), r \sin (\theta))| d \theta, \quad 0 \leq r \leq \pi \sqrt{2} .
$$

In practice are $r$ and $\theta$ discrete and the integration is replaced with a sum. For example the magnitude centered spectrum Fig. 2 a) will result in a graph Fig. 2 b) presenting $A(r)$ calculated from Eq. (2). Finding the radius

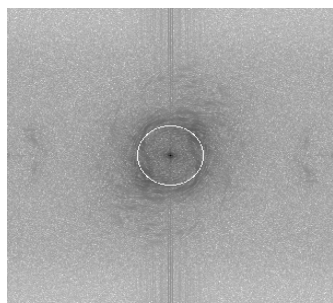

(a)

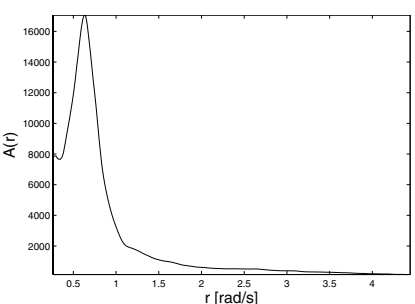

(b)
Figure 2. a) The frequency spectrum and the estimated circle; b) graph of $A(r)$ used for the circle estimation.

corresponding to the highest value of $A(r)$ according to

$$
r_{\max }=\arg \max _{\epsilon<r<\pi \sqrt{2}} A(r),
$$

where $\epsilon$ is a threshold in order to avoid low frequencies. The circle with radius $r_{\max }$ is indicated in Fig. 2 a) and is directly proportional with the dominant spatial frequency in the fingerprint denoted $\Omega_{f}$. The size of local area $\mathcal{L}$ is selected so it enclose multiples of $\Omega_{f}$.

\section{2 $\quad$ Fingerprint Analysis}

After the local area $\mathcal{L}$ size selection a detailed analysis on a local level is performed in the fingerprint image. The dynamic range in each local area is linearly rescaled to the range of $[0,255]$. The window is shifted pixel-by-pixel throughout the fingerprint and the coordinates $\left(\omega_{1}, \omega_{2}\right)$ of each area's $P_{D}$ are extracted from the corresponding magnitude spectrum. The height of each $P_{D}$ is used as an indicator if further investigation is necessary. The FFT process signals with a finite energy therefore a theoretical maximum of the peaks hight can be calculated denoted as $P_{\text {ideal }}$. The ratio between the heights of a local $P_{D}$ and the $P_{\text {ideal }}$ is calculated as

$$
R=\frac{P_{D}}{P_{\text {ideal }}} .
$$

This ratio is in range $0 \leq R \leq 1$ and is illustrated for a typical fingerprint in Fig. 3. Values of $R$ closer to 1 indicates fingerprints regions with high periodic and parallel ridges and vice versa. A threshold is established from which a decision is made whether or not an extra investigation, in form of zooming in or out of the local area, is necessary. If a higher value on $P_{D}$ is found after the additional investigation, the newly found coordinates are chosen. When all coordinates are extracted a smoothing in the polar coordinate domain is performed. 


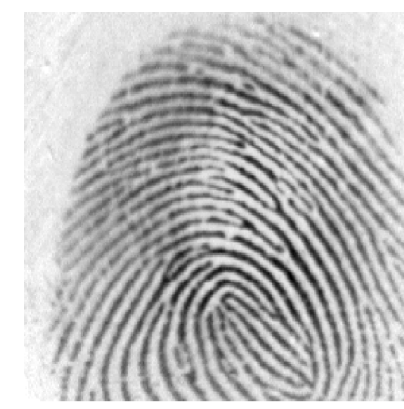

(a)

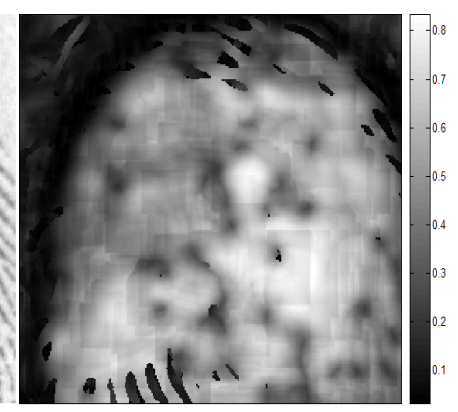

(b)

\section{Figure 3. a) original fingerprint b) analysis of the periodicity in the image in a).}

\subsection{Matched Filter Design and Binariza- tion Process}

When the detailed fingerprint analysis is completed the filters are constructed from the smoothed coordinates obtained in section 2.2 and filtering is performed in the spatial domain. Binarization follows directly after the filtering by thresholding. A post processing is performed after binarization by median filtering the binary fingerprint image.

The matched filters are designed by employing the relationship between the 2-D sinusoidal model and the local fingerprint pattern established in previous part of this paper. Base of the matched filter consists of only one period of the recreated fingerprint pattern by the 2-D sinusoidal model in Eq. (1). The ridge of the base is then cosine tapered to give the final shape of the filter. The interesting property is that the position of the $P_{D}$ in the frequency spectrum will automatically yield the suitable orientation and the size of the filter. The mean of the filter is subtracted in order to obtain a zero value at the DC component.

\section{New Proposals}

In this part improvement to the previously proposed algorithm is described together with some supplementary material.

\subsection{Fingerprint Mask Generation}

Fingerprints usually occupy only a smaller part of the entire fingerprint image. Therefore task of fingerprint mask is to cover all nonrelevant information in the image; only the valid fingerprint part is kept.

Each pixel $\mathbf{x}$ in a fingerprint image $I(\mathbf{x})$ has its significance value $\mathcal{D}_{x}$ measured. The significance value $\mathcal{D}_{x}$ measures how much pixel $\mathbf{x}$ belongs to the binary fingerprint mask $B(\mathbf{x})$. The $\mathcal{D}_{x}$ is calculated with parameters recovered from the frequency spectrum of a local area around each $\mathrm{x}$ denoted as $\mathcal{L}_{x}$ as described in section 2.1. The $\mathcal{D}_{x}$ is computed as

$$
\mathcal{D}_{x}=\frac{P_{D_{x}}}{\mathcal{M}_{x}}
$$

where $P_{D_{x}}$ is the value of dominant peak as mentioned earlier and $\mathcal{M}_{x}$ is a peakenes factor. The $P_{D_{x}}$ and $\mathcal{M}_{x}$ are important features for fingerprint mask generation. Division of $P_{D_{x}}$ with $\mathcal{M}_{x}$ reinforce the final estimation of $B(\mathbf{x})$. The $\mathcal{M}_{x}$ is a mean value calculated as

$$
\mathcal{M}_{x}=\frac{1}{N} \sum_{i_{x}=1}^{N} \frac{P_{i_{x}}}{P_{D_{x}}}
$$

where $N$ is the number of peaks $P_{i_{x}}$ found in the frequency spectrum for each $\mathcal{L}_{x}$. The $P_{i_{x}}$ are found by scanning the frequency spectrum with patches of size $3 \times 3$. If the value in the center of the patch is largest then it is taken as peak $P_{i_{x}}$. The final mask $B(\mathbf{x})$ is generated by thresholding the $\mathcal{D}_{x}$ by threshold $\gamma_{\text {mask }}$

$$
B(\mathbf{x})=\left(\mathcal{D}_{x}>\gamma_{\text {mask }}\right) .
$$

By smoothing the image holding values $\mathcal{D}_{x}$ can further improve the estimation of $B(\mathbf{x})$. All pixels exceeding the threshold are considered as part of the fingerprint, see Fig.4.

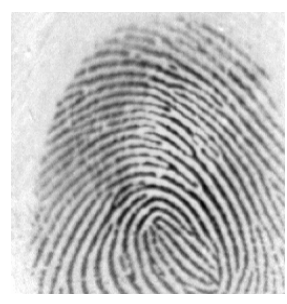

(a)

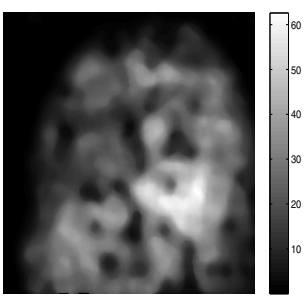

(b) (c)

Figure 4. a) Original fingerprint; b) Estimated $\mathcal{D}_{x}$ for all pixels $\left.\mathbf{x} ; \mathbf{c}\right)$ Fingerprint mask $B(\mathrm{x})$ thresholded by $\gamma_{\text {mask }}=1$.

A scheme to an effictive implementation of peakines $\mathcal{M}_{x}$ estimation is proposed. The pseudo code in the Table 1 illustrates such a scheme. By calculating $\mathcal{M}_{x}$ on-line while 
Table 1. Scheme illustrating an efficient implementation of peakines $\mathcal{M}_{x}$ calculation.

$$
\begin{aligned}
& \begin{array}{l}
\mathcal{M}_{x}=0 \\
P_{D_{x}}=0 \\
\text { for } i_{x}=1,2, \cdots, \mathrm{N} \\
\quad \text { if }\left(P_{i_{x}}>P_{D_{x}}\right) \\
\quad \mathcal{M}_{x}=\left(\mathcal{M}_{x} * \frac{P_{D_{x}}}{P_{i_{x}}}\right)+\frac{P_{D_{x}}}{P_{i_{x}}} \\
\quad P_{D_{x}}=P_{i_{x}}
\end{array} \\
& \quad \text { else } \mathcal{M}_{x}=\mathcal{M}_{x}+\frac{P_{i_{x}}}{P_{D_{x}}} \\
& \quad \text { end } \\
& \text { end } \mathcal{M}_{x}=\frac{\mathcal{M}_{x}}{N}
\end{aligned}
$$

searching for the $P_{D_{x}}$ the first time will result in more time and memory effective algorithm.

\subsection{Post Processing of the Mask}

Rescaling of dynamic ranges in each local area causes the background noise to propagate into the final binarized fingerprints if the original image has a structured background. Quality of binarized fingerprints depends completely on the fingerprint mask. A good fingerprint mask consists of only one large blob outlining the true fingerprint. Analyzing masks of the noisy binarized fingerprints reveals that the masks contain also other usually small blobs. By removing the additional blobs a good noise free binarized fingerprints can be obtained.

Authors propose a post processing step removing the redundant blobs in the fingerprint mask. Labeling algorithm is a method employed on binary images where all connected on-pixels are marked with the same unique tag [7]. In this paper labeling algorithm is employed on the fingerprint mask labeling all blobs with a unique numbers. A computed histogram [4] of the labeled mask provides size of each blob denoted $S_{b l o b}$. The blob with largest size is denoted $S_{b l o b_{M A X}}$ and is used in calculation of ratio denoted $R_{\text {blob }_{i}}$ as following

$$
R_{\text {blobi }_{i}}=\frac{S_{\text {blob }_{i}}}{S_{\text {blob }_{\text {MAX }}}}
$$

where $i$ is the blob index. Value of the threshold $\gamma_{b l o b}$ in

$$
R_{b l o b_{i}}>\gamma_{b l o b}
$$

control the number of remaining largest blobs in the fingerprint mask after thresholding. If $\gamma_{b l o b}=1$ only the largest blob is remaining. In practice the value of the $\gamma_{b l o b}$ is less then one to assure as many parts of the fingerprint is remaining if numerous blobs masks the fingerprint, see Fig. 5.

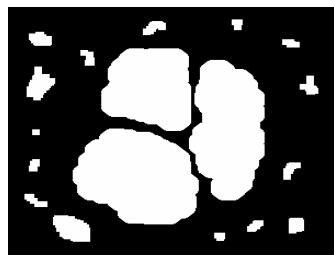

(a)

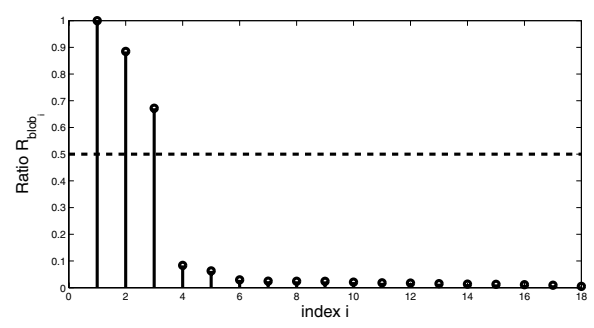

(c)

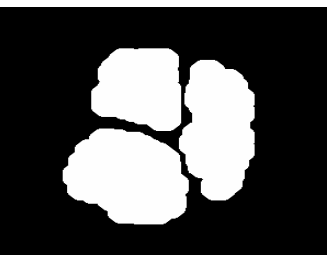

(b)

Figure 5. a) Fingerprint mask; b) Fingerprint mask after thresholding with $\gamma_{b l o b}=0.5$; c) Graph with sorted ratios $R_{b l o b_{i}}$.

\section{Experimental Results}

The previous and the improved adaptive fingerprint binarization algorithms were tested on a fingerprint with structured background. This particular choice of the fingerprint enables the study of improvements introduced in this paper. Fig. 6 presents the original fingerprints together with experimental results. Fig. $6 \mathrm{~b}$ ) and c) illustrates the original results from the previously proposed algorithm [3]. In Fig. $6 \mathrm{~d}$ ) and e) the enhanced results are presented obtained with the improvements presented in this paper.

\section{Conclusions}

In this paper a proposed post processing method employed on the fingerprint mask was introduced. The method demonstrates good capability of removing the redundant noise in the binarized fingerprints. Hence, the final outcome of the extended algorithm is improved in contrast with the previous algorithm. Further, a detailed description of the fingerprint mask generation method has been described. A scheme of effective implementation of the mask generation method has been presented. 


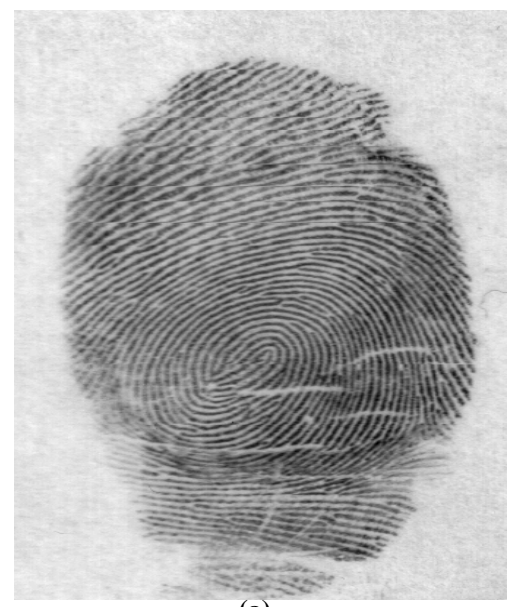

(a)
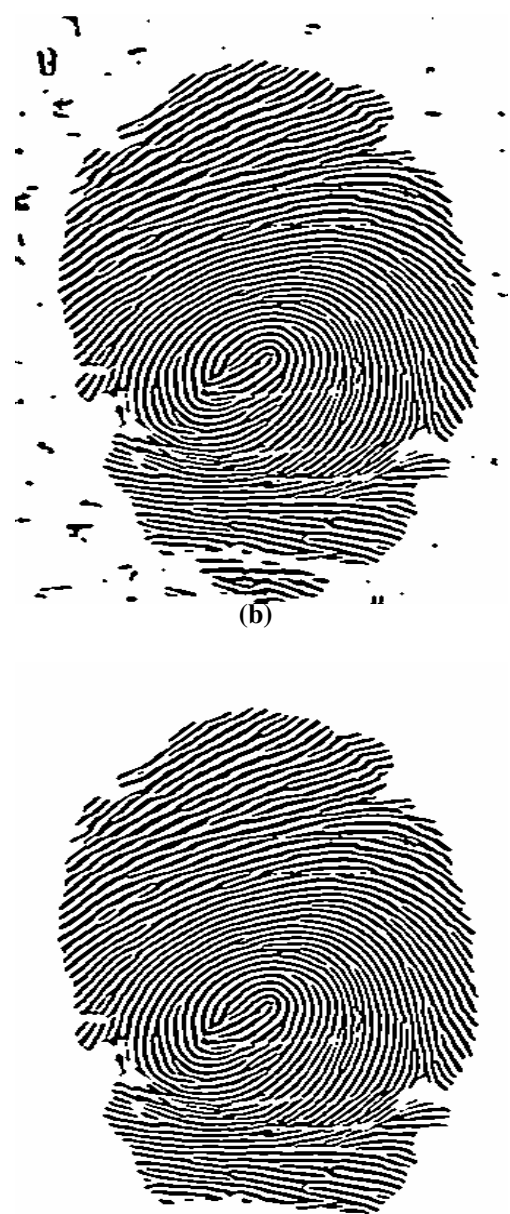

(d)

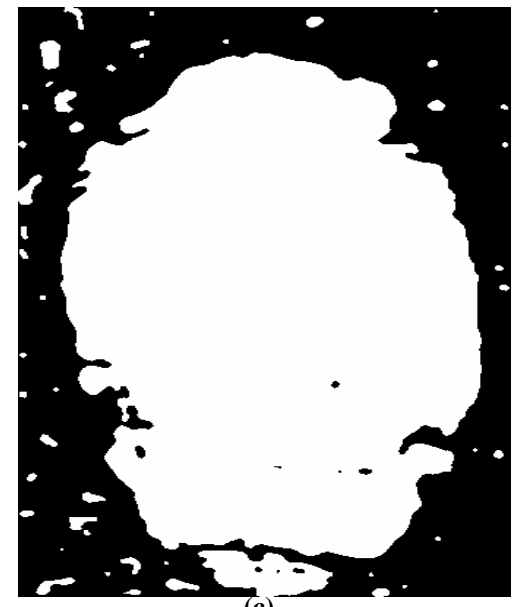

(c)

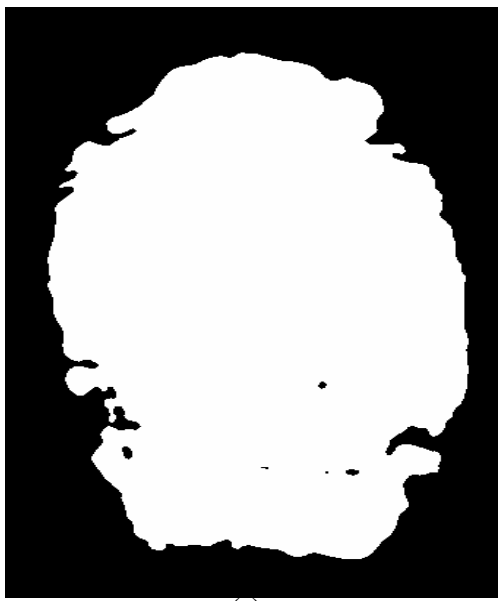

(e)

Figure 6. a) Original fingerprint image; b) and c) are the results from previously proposed algorithm [3]; d) and e) are the improved result by the new modified algorithm.

\section{References}

[1] B.G. Sherlock, D.M. Monro, and K. Millard. Fingerprint enhancement by directional fourier filtering. Vision, Image and Signal Processing, IEE Proceedings-, 141(2):87-94, 1994.

[2] L. Hong, Y. Wan, and A. Jain. Fingerprint image enhancement: Algorithm and performance evaluation. IEEE Transactions on Pattern Analysis and Machine Intelligence, 20(8):777-790, 1998.

[3] J. Ström Bartůněk, M. Nilsson, J. Nordberg, and I. Claesson. Adaptive fingerprint binarization by frequency domain analysis. In Fortieth Asilomar Conference on Signals, Systems \& Computers, pages 598-602, october 2006.

[4] J.S. Lim. Two-Dimensional Signal and Image Processing. Prentice-Hall; 1st edition, 1990. ISBN 0-13-935322-4.

[5] L. O'Gorman and J.V. Nickerson. Matched filter design for fingerprint image enhancement. In Proc. Int. Conf. on Acoustic Speech and Signal Processing, pages 916-919, 1988.
[6] L. O'Gorman and J.V. Nickerson. An approach to fingerprint filter design. Pattern Recognition, 22(1):29-38, 1989.

[7] R.M. Haralick and L.G. Shapiro. Computer and Robot Vision, Volume I. Addison-Wesley,1st edition, 1992. pp. 28-48.

[8] M. Tico, M. Vehvilainen, and J. Saarinen. A method of fingerprint image enhancement based on second directional derivatives. In IEEE International Conference on Acoustics, Speech, and Signal Processing (ICASSP), pages 985-988, 2005. 